\title{
Extensive Pachymeningeal Dissemination of Glioblastoma Mimicking Chronic Subdural Hematoma: A Case Report
}

\author{
Jiwook Lee ${ }^{1}$, Mee-Seon Kim², Young Zoon Kim \\ 'Division of Neurooncology and Department of Neurosurgery, Samsung Changwon Hospital, Sungkyunkwan University \\ School of Medicine, Changwon, Korea \\ ${ }^{2}$ Department of Pathology, Samsung Changwon Hospital, Sungkyunkwan University School of Medicine, Changwon, Korea
}

Received November 13, 2018

Revised January 1, 2019

Accepted January 8, 2019

\section{Correspondence}

Young Zoon Kim

Division of Neurooncology and

Department of Neurosurgery,

Samsung Changwon Hospital,

Sungkyunkwan University

School of Medicine,

158 Paryong-ro, Masanhoewon-gu,

Changwon 51353, Korea

Tel: +82-55-233-5241

Fax: +82-55-233-8040

E-mail: yzkim@skku.edu
Meningeal dissemination (MDS) of glioblastoma is rare, although its incidence might have been underestimated. MDS of glioblastoma has a fatal course. Thus, rapid and precise diagnosis of MDS is important for further palliative treatment. Unfortunately, MDS of glioblastoma could be diagnosed at a delayed time, causing failure to treat patient optimally. Herein, we present a case of a 56-year-old male with MDS of glioblastoma mimicking chronic subdural hemorrhage (CSDH) after head trauma due to slip down. During treatment for $\mathrm{CSDH}$, MDS of glioblastoma was not controlled appropriately. The patient succumbed to MDS of glioblastoma at 9 weeks after the date of diagnosis of CSDH which could be an MDS.

Key Words Glioblastoma; Gliosarcoma; Meningeal dissemination; Mortality.

\section{INTRODUCTION}

Glioblastoma multiforme (GBM) is the most common primary malignant tumor in the central nervous system (CNS) and the most aggressive primary brain tumor in adults, with an incidence of 1.26/100,000 patient-years in Korea [1] and $3.19 / 100,000$ patient-years in the world [2]. Among primary CNS tumors, GBM accounts for 5.3\% of them in Korea [1] and $14.7 \%$ of them in the Unites States [3]. GBM is defined as grade IV according to the World Health Organization (WHO) classification [4]. Despite treatment of choice currently consists of maximal safe surgical resection and postoperative concomitant chemoradiotherapy followed by adjunctive chemotherapy with temozolomide [5], its outcome is still dismal, with

This is an Open Access article distributed under the terms of the Creative Commons Attribution Non-Commercial License (https://creativecommons.org/licenses/by-nc/4.0) which permits unrestricted non-commercial use, distribution, and reproduction in any medium, provided the original work is properly cited.

Copyright $\odot 2019$ The Korean Brain Tumor Society, The Korean Society for NeuroOncology, and The Korean Society for Pediatric Neuro-Oncology a median survival time of 14.6 months [6].

GBM often occurs in the supratentorial white matter including the corpus callosum. It usually spreads along white matter tracts [7]. Ependymal, subpial, and cerebrospinal fluid (CSF) metastases into the subarachnoid space are events often seen in terminal patients. Leptomeningeal dissemination (LMD) of GBM is known to be rare. However, its incidence might have been underestimated in clinical practice. Clinically, the reported incidence of LMS from malignant glioma had been $2 \%$ [8] and 3.1\% [9]. However, autopsy studies have shown higher incidence of leptomeningeal seeding than those in the neurooncological practice $[10,11]$. One reason for such discrepancy may be that rapid disease progression can result in loss of chance to survey LMD of GBM. Since the prognosis of GBM patients with LMD is bleak often with fatal outcome, early and precise diagnosis of LMD is important to care for such patients.

Herein, we present a GBM patient with extensive meningeal dissemination (MDS) mimicking chronic subdural hemor- 
rhage $(\mathrm{CSDH})$ in CT scan performed after head trauma, which makes the best treatment delayed for the MDS of GBM. Also, we are reviewing the literature focused on GBM with MDS.

\section{CASE REPORT}

A 56-year-old male was admitted into the Neurosurgical Department via out-patient clinic due to progressive hemiparesis of grade 3 weakness on the left side for two weeks. On a previous regular check for personal health performed four months earlier at our institute, magnetic resonance (MR) image showed a small focus with high signal intensity in fluid attenuated inversion recovery (FLAIR) image on the right precentral gyrus of the frontal lobe. He had no focal neurological symptoms or signs. The lesion did not have enhancement with gadolinium in T1WI at that time. However, at the present admission, MR image showed single nodule at size of $1 \mathrm{~cm} \times 1$ $\mathrm{cm}$ with rim enhancement on the previous area showing high signal intensity in FLAIR image (Fig. 1).

GBM was diagnosed histopathologically based on navigation-guided frameless stereotactic biopsy. Molecular and genetic analyses had the following results: O6-methyl guanine DNA methyltransferase (MGMT) promoter, unmethylated; isocitrate dehydrogenase 1 , wildtype; $1 \mathrm{p} 19 \mathrm{q}$, not deleted; al- pha-thalassemia (ATRX), retained expression; amplification for p53 and epidermal growth factor receptor (EGFR), positive; mitotic figures of 15/10 high power field (HPF); CD133, positive; phosphatase and tensin homolog (PTEN), negative; glial fibrillary acidic protein (GFAP), positive; and Ki67 14\%. Then he underwent concurrent chemoradiotherapy with temozolomide for six weeks followed by six cycles of adjuvant treatment with temozolomide using Stupp's regimen. There was no progression at the 12-months follow-up in MR images. His neurological condition did not get worsen either. His physical condition was tolerable.

However, the MR image which was performed regularly by 6 months' interval obtained at 2 years after the initial diagnosis illustrated an aggressive progression with heterogeneous enhancement and extensive peritumoral edema (Fig. 2). His headache and motor weakness got worse gradually and did not respond to high dose corticosteroid. His motor function worsened to grade 1 on the left side. Therefore, craniotomy and gross total resection were performed to reduce the tumor burden and improve symptoms. Histopathological features showed two biphasic patterns in tumor of epithelioid glial component and cellular spindle mesenchymal component, leading to the diagnosis of gliosarcoma (Fig. 3). After surgical resection, the headache was relieved and the hemiparesis on the
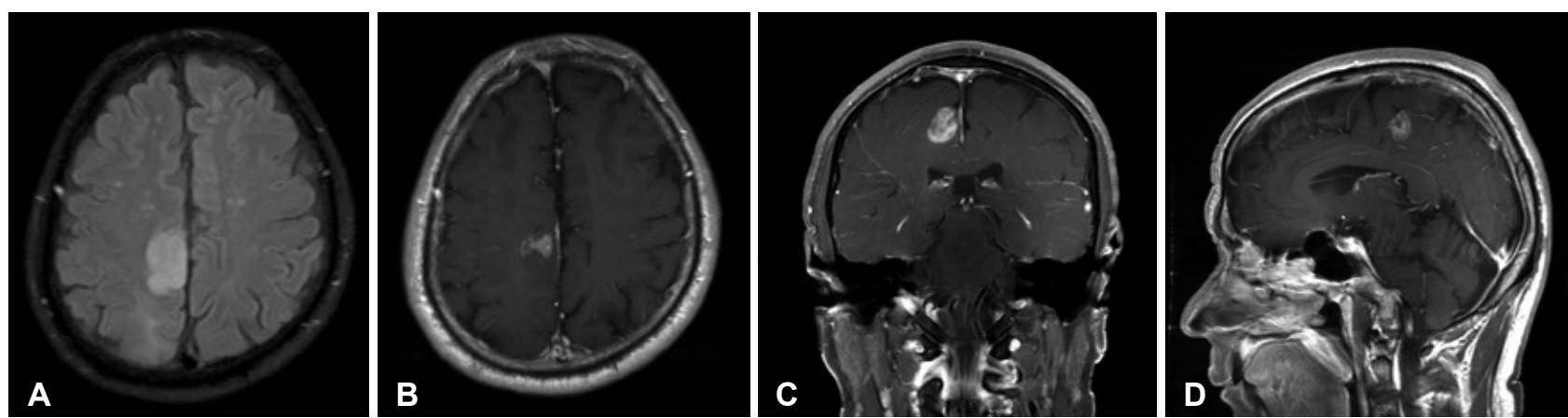

Fig. 1. The second MR image at the time of occurrence of progressive hemiparesis on the left side showing increased size of the previous area with high signal intensity in fluid attenuated inversion recovery image $(A)$, and nodular rim enhancement at size of $1 \mathrm{~cm} \times 1 \mathrm{~cm}$ showing in T1WI axial (B) coronal (C), and sagittal images (D) in the right frontoparietal area.
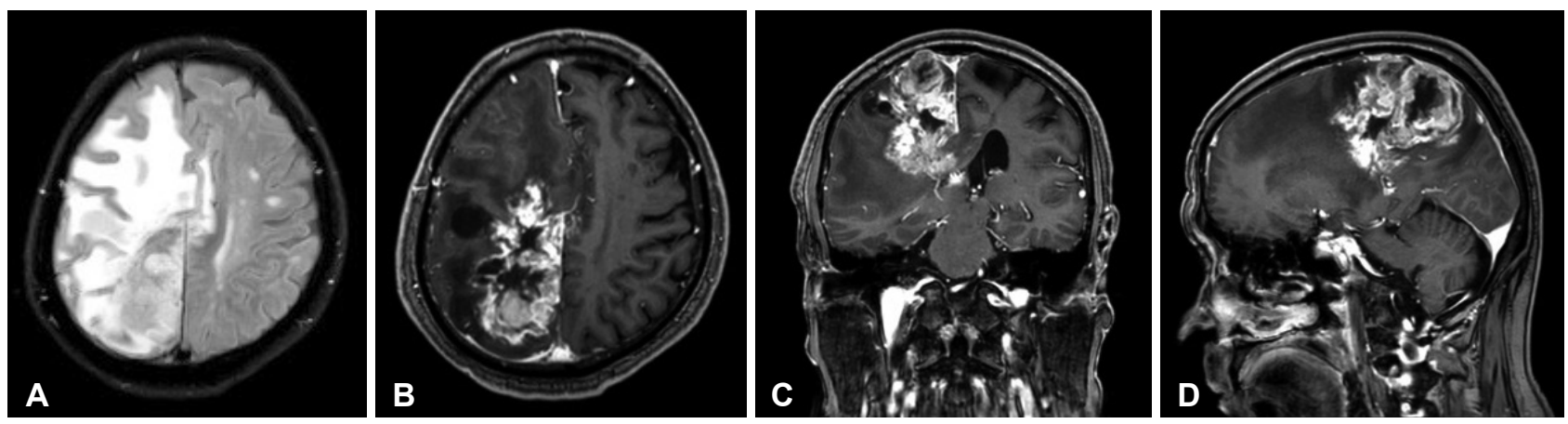

Fig. 2. MR image for regular follow-up showing more extensive peritumoral edema in the fluid attenuated inversion recovery image (A), the low signal intensity suggesting necrosis, irregular shaped unevenly thicken walled mass with internal necrosis, and heterogeneous enhancement on axial (B), coronal (C), and sagittal images (D) in the right frontoparietal area. 
left side was improved to grade 4 . He started to take metronomic chemotherapy with daily treatment of temozolomide at dose of $50 \mathrm{mg} /$ body surface area.

Four months later, he had a mild head trauma due to slip down which made him suffer from chronic headache and decrease of mentality. The laboratory study showed no abnor- mal findings in the complete blood count, liver function test, bleeding times and coagulation test, but hypercholesterolemia (total cholesterol of $255 \mathrm{mg} / \mathrm{dL}$ and triglyceride of $455 \mathrm{mg} /$ dL) with hyperglycemia (HbA1c of $8.7 \%$ ). In CT scan, fluid collection with low density under the dura mater on the right frontotemporoparietal area was shown (Fig. 4A). Straw-col-
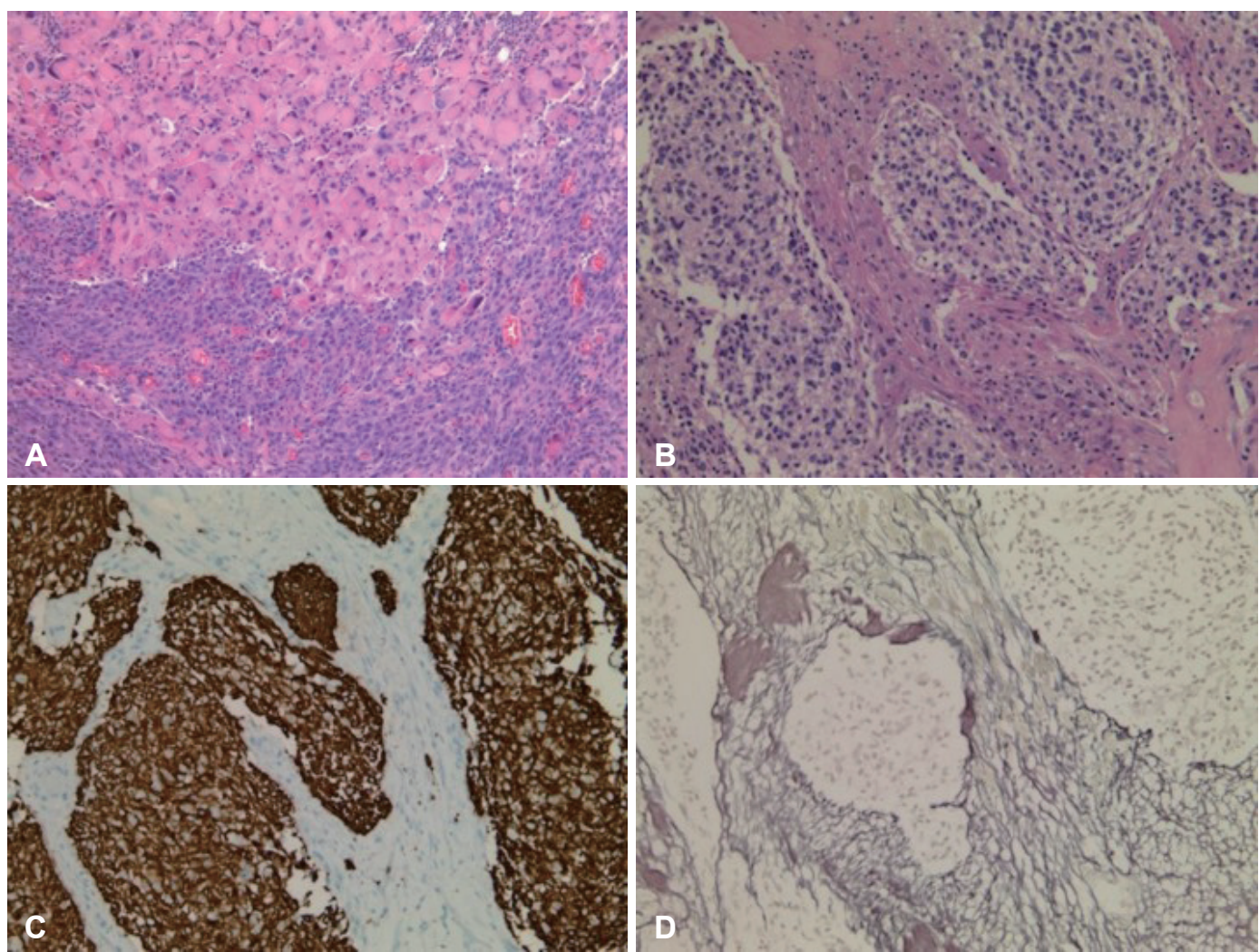

Fig. 3. Histopathological features showing two biphasic patterns in tumor of epithelioid glial component (upper lane) and cellular spindle mesenchymal component (bottom lane); (H\&E staining, $\times 20)(A)$. The glial component surrounded by elongated mesenchymal component and the glial area is composed of mild pleomorphic astrocyte (H\&E staining, $\times 20)(B)$, leading to the diagnosis of gliosarcoma. Immunohistochemistry for GFAP demonstrates positivity in glial component $(\times 20)(C)$, and the biphasic pattern shows strong reticulin positivity in mesenchymal areas with reticulin negative glial areas (reticulin stain, $\times 20)(D)$. H\&E: hematoxylin and eosin.
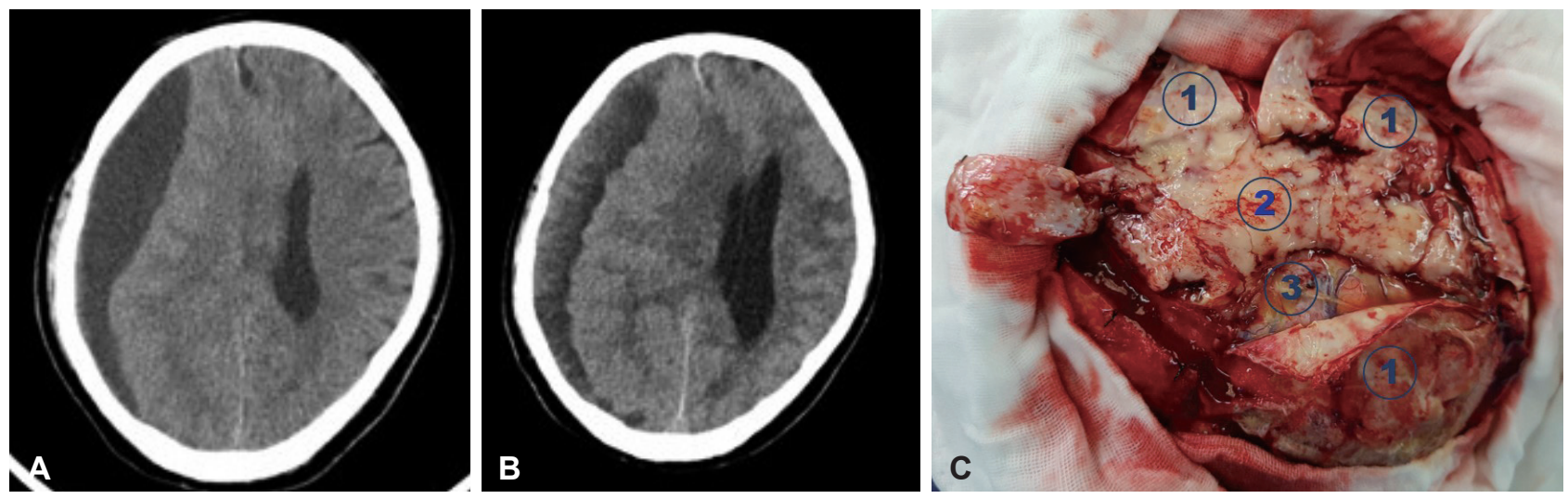

Fig. 4. The noncontrast CT scan showing a newly developed subdural fluid collection which is suggestive hematoma at the right hemisphere (A). Repeated CT scan without contrast showing the lining pattern of soft tissue density as well as fluid collection at the right hemisphere (B). Intraoperative findings (C) show that thickened dura matter with attachment of the tumors (1)), whitish colored tumor with a fragile texture and relatively poor vascularity (2)), and the relatively well preserved arachnoid membrane beneath the tumor without destruction nor invasion by the tumor (3). 
ored fluid was drained via the burrhole trephination. The fluid had high viscosity and looked like mucoid fluid. After drainage, the patient's headache subsided moderately. However, the low density remained minimally in the follow-up CT scan which was checked 4 weeks after the first burrhole drainage. And progressive decrease of mentality to stupor with thickened low-density area in the CT scan. Thus, he underwent a second burrhole trephination for drainage of CSDH. However, no fluid was drained. Therefore, craniotomy was performed to remove the subdural fluid collection (Fig. 4B). During operation, there was no fluid collection but a mass covering the whole hemisphere on the right side of frontotemporoparietal lobe. The whitish-colored tumor had a fragile texture and relatively poor vascularity, was attached at the whole pachymeninges of the right hemisphere (Fig. 4C), and easily detached by bipolar coagulators. However, the arachnoid membrane beneath the tumor was not destructed nor invaded by the tumor but relatively well preserved. The mass was removed as much as possible, and histological characteristics of the glioblastoma were changed into more aggressive and more malignant biology as follows: 1p19q non-codeleted; MGMT nonmethylated; CD133, strong positive; PTEN, negative; GFAP, strong positive; EGFR, 3+; ATRX, retained expression; p53 with about $35 \%$; Ki67 with about 50\%; mitotic figures of $85 / 10$ HPF.

His family members were reluctant to let him undergo further palliative therapies such as re-irradiation or chemotherapy despite worsening features in patient's condition and CT scan. He was succumbed to the disease at 4 weeks after the last craniotomy. His overall survival was 32 months. He died at 9 weeks after diagnosis of LMD based on the CT scan. Informed consent was waived due to its retrospective nature and minimal risk for harm to patient. And this report was conducted according to guidelines of the Declaration of Helsinki for biomedical research.

\section{DISCUSSION}

Meningeal spread including ependymal, subpial, and CSF metastases into the subarachnoid and subdural space is not commonly encountered in the clinical field of treating GBM and even events seen in terminal patients. Symptomatic CSF seeding of intracranial GBM is also rare, accounting for only $2 \%$ of cases in the largest study of 600 patients [12]. That study also showed that CSF seeding occurred mostly in the late stage of GBM [12]. However, it is still unknown whether lengthier survival may lead to greater chance of CSF seeding.

In fact, glioma dissemination, especially invasion into the leptomeninges, has been largely studied. There are growing studies reporting potential risk factors and possible mecha- nisms for leptomeningeal spread of GBMs. Among them, the anatomical location of GBM is the most important risk factor. It is well-known that there is a particular relationship between GBM and the subventricular zone (SVZ) neural stem cells region [13]. GBMs in contact with the SVZ are very likely to be multifocal at diagnosis and recur a distance from the initial lesion [13]. Additionally, ventricular entry at operation, repeated tumor resection, male sex, ependymal invasion, fissuring of the ependyma due to hydrocephalus, depressed immune function after radiotherapy and chemotherapy, and fragmentation of the tumor in contact with CSF are significant factors associated with increased incidence of MDS [14].

In terms of molecular biology, a number of factors and mechanisms for MDS of GBM have been implicated, including CD133-postive stem cells as possible initiators of metastasis. Matrix metalloproteinases involved in enzymatic degradation of the extracellular matrix also appear to play a key role in the progression of various tumors. Mutations in tumor suppressor gene PTEN as well as gains at the $1 \mathrm{p} 36$ chromosomal region have also been preferentially found in disseminated glioma [15-17]. For histopathological diagnosis, some studies have suggested that the degree of astrocytic differentiation of tumor cells seen in GFAP-immunohistochemistry and MIB-1 labelling indexes are potential markers of invasiveness [15,18, 19]. However, the present case did not show $\mathrm{CD} 133$ positivity nor loss of PTEN, although he showed low expression level of GFAP in immunohistochemical staining with high Ki67 index (about 40\%). Since these molecular features are from a single case, they may not reflect all cases. Further comprehensive studies are essential for establishment of predictive role of molecular studies.

Treatment for MDS of GBM is chiefly palliative due to its fatal prognosis. The mean survival time between diagnosis of MDS and death is approximately 2 to 3 months [20,21]. Radiotherapy is the most common treatment of choice, with a total dose of 25-40 Gy. It may provide pain relief and some improvements in neurological function. However, it may not offer survival benefits [22]. Intravenous or intrathecal chemotherapy has not found to be very useful for improving the overall survival of such patients either. Repeated surgical resection may be attempted if there is a symptomatic, large metastatic deposit causing cord compression. However, usually MDS is not amenable to surgery due to diffuse nature of the disease [22]. In the present case, we performed surgical resection for the subdural located large mass covering the right hemisphere. However, the patient's neurological status or survival was not improved at all.

In the present case, we misdiagnosed MDS as CSDH due to obvious history of head trauma and clinical manifestation. However, several diseases must be differentiated from MDS 
in CT scan and MR image. The first of such diseases that need to be differentiated is meningeal metastasis of systemic cancer such as breast cancer and prostate cancer [23]. On non-contrast-enhanced CT images and MR image, it is difficult to differentiate CSDH from meningeal metastasis of systemic cancer. Dural metastases appearing as relatively dense lesions and hemorrhagic hyperdensities may be found. On MR, most masses are hypointense to grey matter in T1WI and hyperintense in T2WI with possible hemorrhagic signal as in any metastasis. The second of such diseases that need to be differentiated from MDS is meningiomas, especially the en plaque type which is a common primary adult intracranial tumor [23]. In nonenhanced CT, the mass is sharply circumscribed in most cases $(70-75 \%)$ while it is hyperdense with calcification in 20$25 \%$ of cases. Necrosis, cysts, and hemorrhage occur in $8-23 \%$ of cases. The third is neurosarcoidosis, a multisystem inflammatory disease characterized by non-caseating epitheloid-cell granulomas of unknown etiology with granulomatous leptomeningitis or dural-based mass as the most common gross pathological features [23]. Therefore, physicians must differentiate MDS from other pathologic conditions even if the cause is obvious such as the presenting case. Despite the MR image is superior to CT scan for the differentiation of these pathological condition, we did not check the MR image unfortunately. The omitting check of MR image could be one of reasons doing our misdiagnosis.

In summary, we experienced a case of extensive MDS of GBM misdiagnosed as CSDH due to obvious history of head trauma. Here we report this case with literature review. Although the dismal outcome of MDS of GBM makes physician and patients difficult to treat this disease, further comprehensive study is needed to improve the outcome of patients with MDS of GBM.

\section{Conflicts of Interest}

The authors have no potential conflicts of interest.

\section{Acknowledgments}

We appreciate the two radiologist, Young Min Kim, M.D., and Sunwoo Mi-Ok, M.D. for the review of radiographic features of the patient.

\section{REFERENCES}

1. Dho YS, Jung KW, Ha J, et al. An updated nationwide epidemiology of primary brain tumors in Republic of Korea, 2013. Brain Tumor Res Treat 2017;5:16-23.

2. Louis DN, Perry A, Burger P, et al. International Society of Neuropathology--Haarlem consensus guidelines for nervous system tumor classification and grading. Brain Pathol 2014;24:429-35.

3. Ostrom QT, Gittleman H, Truitt G, et al. CBTRUS statistical report: primary brain and other central nervous system tumors diagnosed in the United States in 2011-2015. Neuro Oncol 2018;20(suppl_4):iv1-86.

4. Louis DN, Perry A, Reifenberger G, et al. The 2016 World Health Or- ganization Classification of Tumors of the Central Nervous System: a summary. Acta Neuropathol 2016;131:803-20.

5. Preusser M, de Ribaupierre S, Wöhrer A, et al. Current concepts and management of glioblastoma. Ann Neurol 2011;70:9-21.

6. Stupp R, Hegi ME, Mason WP, et al. Effects of radiotherapy with concomitant and adjuvant temozolomide versus radiotherapy alone on survival in glioblastoma in a randomised phase III study: 5-year analysis of the EORTC-NCIC trial. Lancet Oncol 2009;10:459-66.

7. Kumar R, Jain R, Tandon V. Thalamic glioblastoma with cerebrospinal fluid dissemination in the peritoneal cavity. Pediatr Neurosurg 1999;31: 242-5.

8. Salazar OM, Rubin P. The spread of glioblastoma multiforme as a determining factor in the radiation treated volume. Int J Radiat Oncol Biol Phys 1976;1:627-37.

9. Macdonald DR, Cascino TL, Schold SC Jr, Cairncross JG. Response criteria for phase II studies of supratentorial malignant glioma. J Clin Oncol 1990;8:1277-80.

10. Bryan P. CSF seeding of intra-cranial tumours: a study of 96 cases. Clin Radiol 1974;25:355-60.

11. Saito R, Kumabe T, Jokura H, Shirane R, Yoshimoto T. Symptomatic spinal dissemination of malignant astrocytoma. J Neurooncol 2003;61: 227-35.

12. Vertosick FT Jr, Selker RG. Brain stem and spinal metastases of supratentorial glioblastoma multiforme: a clinical series. Neurosurgery 1990; 27:516-21.

13. Lim DA, Cha S, Mayo MC, et al. Relationship of glioblastoma multiforme to neural stem cell regions predicts invasive and multifocal tumor phenotype. Neuro Oncol 2007;9:424-9.

14. Lawton CD, Nagasawa DT, Yang I, Fessler RG, Smith ZA. Leptomeningeal spinal metastases from glioblastoma multiforme: treatment and management of an uncommon manifestation of disease. J Neurosurg Spine 2012;17:438-48.

15. Kato H, Fujimura M, Kumabe T, Ishioka C, Kanamaru R, Yoshimoto T. PTEN gene mutation and high MIB-1 labeling index may contribute to dissemination in patients with glioblastoma. J Clin Neurosci 2004;11: 37-41.

16. Korshunov A, Sycheva R, Golanov A, Pronin I. Gains at the 1p36 chromosomal region are associated with symptomatic leptomeningeal dissemination of supratentorial glioblastomas. Am J Clin Pathol 2007;127: 585-90.

17. Sato A, Sakurada K, Kumabe T, et al. Association of stem cell marker CD133 expression with dissemination of glioblastomas. Neurosurg Rev 2010;33:175-83.

18. Arita N, Taneda M, Hayakawa T. Leptomeningeal dissemination of malignant gliomas. Incidence, diagnosis and outcome. Acta Neurochir (Wien) 1994;126:84-92.

19. Onda K, Tanaka R, Takahashi H, Takeda N, Ikuta F. Cerebral glioblastoma with cerebrospinal fluid dissemination: a clinicopathological study of 14 cases examined by complete autopsy. Neurosurgery 1989;25:53340.

20. Lomax AJ, Yannakou CK, Rosenthal MA. Spinal cord metastasis in a patient treated with bevacizumab for glioblastoma. Target Oncol 2013; 8:153-5.

21. Bae JS, Yang SH, Yoon WS, Kang SG, Hong YK, Jeun SS. The clinical features of spinal leptomeningeal dissemination from malignant gliomas. J Korean Neurosurg Soc 2011;49:334-8.

22. Grah JJ, Katalinic D, Stern-Padovan R, et al. Leptomeningeal and intramedullary metastases of glioblastoma multiforme in a patient reoperated during adjuvant radiochemotherapy. World J Surg Oncol 2013;11:55.

23. Taschner CA, Brendecke S, Weyerbrock A, Egger K, Prinz M. Freiburg neuropathology case conference: widespread mass lesions after resection of a glioblastoma multiforme. Clin Neuroradiol 2012;22:375-80. 